\title{
Identification of sex-specific genetic associations in response to opioid analgesics in a White, non-Hispanic cohort from Southeast Minnesota
}

\author{
Guilherme S. Lopes ${ }^{1 凶}{ }^{凶}$, Jaime L. Lopes ${ }^{2}$, Suzette J. Bielinski ${ }^{3}$, Sebastian M. Armasu ${ }^{3}$, Ye Zhu ${ }^{3}$, Dana C. Cavanaugh ${ }^{4}$, Ann M. Moyer (iD ${ }^{3}$, \\ Debra J. Jacobson ${ }^{3}$, Liwei Wang ${ }^{3}$, Ruoxiang Jiang ${ }^{3}$, Jennifer L. St. Sauver ${ }^{3}$ and Nicholas B. Larson ${ }^{3}$
}

(c) The Author(s) 2022

\begin{abstract}
The study of sex-specific genetic associations with opioid response may improve the understanding of inter-individual variability in pain treatments. We investigated sex-specific associations between genetic variation and opioid response. We identified participants in the RIGHT Study prescribed codeine, tramadol, hydrocodone, and oxycodone between 01/01/2005 and 12/31/2017. Prescriptions were collapsed into codeine/tramadol and hydrocodone/oxycodone. Outcomes included poor pain control and adverse reactions within six weeks after prescription date. We performed gene-level and single-variant association analyses stratified by sex. We included 7169 non-Hispanic white participants and a total of 1940 common and low-frequency variants (MAF $>0.01$ ). Common variants in MACROD2 (rs76026520), CYP1B1 (rs1056837, rs1056836), and CYP2D6 (rs35742686) were associated with outcomes. At the gene level, FAAH, SCN1A, and TYMS had associations for men and women, and NAT2, CYP3A4, CYP1A2, and $S L C 22 A 2$ had associations for men only. Our findings highlight the importance of considering sex in association studies on opioid response.
\end{abstract}

The Pharmacogenomics Journal (2022) 22:117-123; https://doi.org/10.1038/s41397-022-00265-9

\section{INTRODUCTION}

Opioids are among the most commonly prescribed medications in the United States, with 51 opioid prescriptions per 100 persons filled in 2018 [1]. Despite the widespread use of opioids, there are important individual differences in opioid response in terms of pain relief, adverse reactions, and addiction [2-4]. Some of the variability is attributable to genetic factors, which are known to contribute to significant variability in pain perception and opioid metabolism $[5,6]$. Genetic factors have explained about $30-76 \%$ of the inter-individual variability in opioid response in animal models [7]. To date, genetic association studies have reported over 150 genes associated with chronic pain conditions [5], some of which are also implicated in the risk of poor pain control and adverse reactions due to opioid analgesics [8]. For example, the cytochrome P450 2D6 enzyme is involved in prodrug conversion of opioids to their active metabolites. Specifically, genetic variation in CYP2D6 directly affects CYP2D6 enzyme activity [9], which can predict a person's likelihood to experience poor pain control and adverse reactions [10]. However, despite much progress in the field of individualized medicine, opioidbased treatments still have a high degree of inter-individual response variability attributable to their pharmacokinetic and pharmacodynamic properties [5]. Identifying novel genetic risk factors associated with opioid metabolism may help explain this variability and lead to more optimized treatment regimens.

In addition to genetic factors, response to opioid medications has been shown to vary by sex. For example, women are more likely to experience adverse reactions after opioid administration (e.g., nausea and vomiting) [11, 12]. Genetic loci associated with pain perception plays an important role in treatment effectiveness [5, 13], and some genetic loci associated with pain perception have also been demonstrated to be sex specific [14]. However, few pharmacogenomics (PGx) studies on pain perception and opioid metabolism have stratified results by sex. Among those studies, even fewer focused on specific outcomes of opioid medications, such as pain control and adverse reactions, with mixed results [15-17].

Together, these studies show that only a limited number of genetic variants have been identified as important in responses to opioid medications, and even fewer studies have examined the role of sex and genetic variation interactions. Identification of sex-specific genetic associations with opioid response may help clinicians to better individualize pain treatment, thereby optimizing effectiveness and reducing adverse reactions related to opioid use. To address these questions, we investigated sex-specific associations between genetic variation captured by a PGx sequencing panel and response to four commonly prescribed opioids in a large sample of individuals.

\section{METHODS}

Study participants

Details of the RIGHT 10K Study have been previously reported [18-20]. Briefly, 10074 participants in the Mayo Clinic Biobank were invited and agreed to participate in a study of pre-emptive sequencing to understand

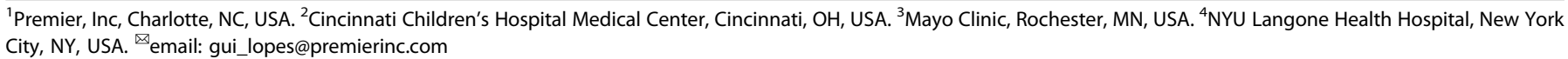

Received: 30 March 2021 Accepted: 10 January 2022

Published online: 31 January 2022 
the effect of variation in genes related to drug metabolism on drug outcomes. PGx sequencing results were deposited into the electronic health record (EHR) for use in clinical care. At the time of enrollment, participants in the RIGHT study provided written informed consent to allow their PGx genetic data and EHR data to be used for research in future studies. This study was approved by Institutional Review Boards (IRB) at the Mayo Clinic and Olmsted Medical Center, and was conducted in accordance with the Declaration of Helsinki.

\section{Opioid prescription}

The Rochester Epidemiology Project (REP) is a medical records-linkage system connecting collaborating clinics, hospitals, and other medical facilities in Minnesota and Wisconsin, United States, and includes data of community members who have agreed to share their medical records for research [21]. The REP captures medical data from all participants in the RIGHT Study. We therefore utilized the REP research infrastructure to identify all participants in the RIGHT Study who were prescribed formulations containing codeine, tramadol, hydrocodone, or oxycodone between 1/1/2005 and 12/31/2017. We focused on these four opioids because they are commonly prescribed in the general population. We used RxNorm - a national database of normalized names for clinical drugs-to identify all codes for prescriptions that included one of the four opioids. We excluded formulations intended primarily to treat cough (e.g., guaifenesin/codeine). Participants prescribed one of the four opioids between $1 / 1 / 2005$ and 12/31/2005 who also had an opioid prescription between $1 / 1 / 2004$ and 12/31/2004 were excluded to focus on initiators of opioid use. Some formulations included nonsteroidal anti-inflammatory drugs (e.g., lbuprofen/Oxycodone), which may contribute to increased pain relief beyond the effects of the opioid. Our study focused on formulations containing opioids. A complete list of formulations considered is shown in Supplemental Table 1.

\section{Outcomes}

Opioid-related outcomes included a binary indication (yes/no) of poor pain control (e.g., clinical note indicating need to change dose or type of drug due to uncontrolled pain) and adverse reactions related to medication use (e.g., nausea, itching, constipation) attributed to the prescription within 6 weeks after each opioid prescription date. To identify potential opioid-related outcomes, EHRs were initially screened by natural language processing techniques using MedTagger [22]. Specifically, sentences including each opioid's ingredient or trade names and keywords related to poor pain control or presence of adverse reactions due to opioid use were extracted from the clinical notes. All sentences were then reviewed by trained abstractors (JLS, DCC, and YZ), and classified as poor pain control (yes/no) or adverse reaction (yes/no). For example, the sentence "Patient has been taking Ultram but continues to complain of severe neck pain" was considered an indication of poor pain control associated with tramadol. This process is summarized in Supplemental Fig. 1. All records were reviewed blinded to the patients' genetic information. Outcomes that could not be associated with a specific opioid prescription were not included (e.g., the sentence "feels nausea due to medication" written within 6 weeks of an opioid prescription does not specify whether this adverse reaction is due to the prescribed opioid or to another medication).

\section{PGx sequencing}

PGx sequencing was performed by the Clinical Laboratory Improvement Amendments-certified and College of American Pathologists-accredited Baylor College of Medicine's Human Genome Sequencing Center Clinical Laboratory using the Pharmacogenomics Research Network (PGRN)-Seq v3 targeted capture sequencing panel. Details of the PGx sequencing in the RIGHT Study have been previously reported [18-20]. To potentially improve downstream quality control analyses that traditionally rely on genome-wide data, we additionally performed genome-wide genotyping of off-capture regions. Additional variant level annotation was assigned to all called variants using BioR [23], including output from Clinical Annotation of Variants (CAVA) [24].

\section{Quality control}

Variants were excluded from analysis if the variant call rate among all sequenced participants was $<0.95$. Despite the limited amount of genetic variation captured by the PGRN-Seq v3 assay, we attempted preliminary evaluation of population substructure and relatedness using both on- and off-capture genotype calls. Population structure was evaluated via LASER using Fast and Robust Ancestry Coordinate Estimation and the Human Genome Diversity Project reference panels, while relatedness was assessed using PREST. Results were largely consistent with self-reported race/ethnicity (Supplemental Fig. 2), and thus we applied the latter as the basis for subsetting to an ancestrally homogenous subgroup of RIGHT participants. Results from PREST indicated evidence of substantial relatedness present, although identity-by-descent estimates were too noisy to confidently resolve genetic relationships (Supplemental Fig. 3). To fully leverage all available data, we considered mixed-model genetic association analysis approaches that can accommodate cryptic relatedness and latent population substructure using an estimated genetic relatedness matrix (GRM). For variants among self-reported non-Hispanic whites, we estimated the minor allele frequency (MAF) and conducted Hardy-Weinberg equilibrium (HWE) for variants with empirical MAF $>0.05$. Given that the presence of cryptic relatedness can lead to HWE test Type I error inflation, we considered significant deviation from HWE at a conservative $p$ value $<1 \times 10^{-5}$ and excluded such variants from further single-variant analysis.

\section{Statistical analyses}

Patient characteristics were summarized using counts and percentages for categorical variables, and median and quartiles for continuous variables. Sex was retrieved from the EHR. Age was calculated for each date of opioid's prescription.

To improve sample size in analysis, we performed analyses by related drug groups based on participants who were prescribed codeine and/or tramadol or hydrocodone and/or oxycodone. These groupings follow previous research $[16,25]$ and are based on drug similarities in pharmacodynamic profiles. For example, hydrocodone and oxycodone are phenanthrenes, and patients report similar pain relief and adverse reactions to hydrocodone and oxycodone, including nausea, vomiting, itching, and drowsiness [26]. In contrast, codeine, which contains the 6-hydroxyl groups, is associated with more nausea and hallucinations than hydrocodone and oxycodone, which do not have the 6-hydroxyl groups [27]. In addition, the difference in affinity for the opioid receptor between parent drug and active metabolite is not as large for hydrocodone and oxycodone compared to tramadol and codeine. Under this analysis design, participants could be included twice in an analysis if they were prescribed drugs from different groupings.

For each single-SNP association analysis, autosomal variants with an empirical MAF $>0.01$ among analyzed participants were considered for testing, and analyses were performed both combined across and stratified by sex. To account for residual population stratification and cryptic relatedness, we applied generalized linear mixed-effects (GLMM) modeling using the $R$ package GMMAT [28]. GMMAT applies an estimated GRM to account for genetic similarity across study participants. A sparse GRM was generated such that entries $<0.125$ were truncated to zero. We also allowed for a random intercept to account for multiple observations per participant with multiple drug exposures in a given drug group. A multivariable logistic GLMM was used to model associations between each variant in the risk of poor pain control or adverse reactions to each group of opioids. Testing was performed using the GMMAT score test under an additive genetic model while additionally adjusting for age at prescription, sex, and prescribed drug (e.g., codeine [yes/no] and tramadol [yes/no] for the codeine/tramadol group). The direction of effect was determined based on the sign of the score test statistic. Variant associations were declared to be statistically significant at a false discovery rate (FDR) threshold of $<0.05$ using the Benjamini-Hochberg approach, which was also used to compute FDRadjusted $p$ values ( $q$ values). FDR control was conducted stratified by each analysis ( 2 drug groups $\times 3$ cohorts $\times 2$ outcomes $=12$ total analyses). Suggestive variant associations were also highlighted at a $p$ value $<0.001$.

Additionally, we conducted a gene-level analysis to investigate the aggregate effects of common and rare variants for all directly sequenced genes. Specifically, we applied the efficient hybrid test SMMAT-E implemented in GMMAT under the same models described above for single-variant association analysis, restricted to variants located within the respective gene boundaries. Default MAF-based beta weights were applied to place more weight on rarer variation. Given the relatively small number of genes and likely weak genetic correlation across genes, we adopted a simple Bonferroni-corrected alpha of 0.05 to define statistical significance of gene-based testing, where the correction factors were based on total number of genes tested and sex strata (77 genes by 2 sexes). We conducted analyses including a filtered subset of identified variants based on CAVA impact values of "moderate" or "high", as these single nucleotide polymorphisms (SNPs) are more likely to affect protein function. To aid in 
Table 1. Patient characteristics by sex among patients prescribed codeine/tramadol ( $n=3636)$ and hydrocodone/oxycodone ( $n=6649)$.

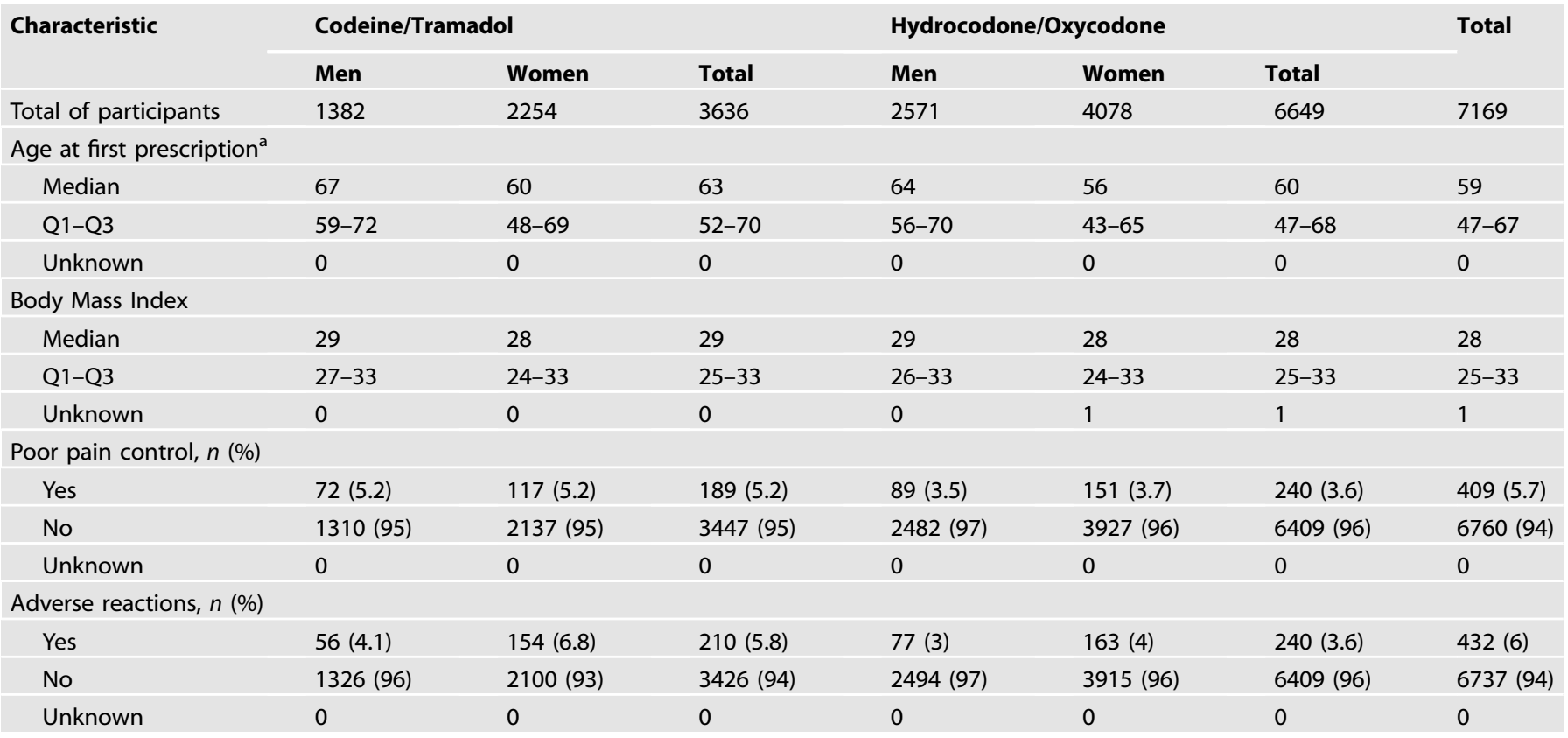

Note: Percentages are by column.

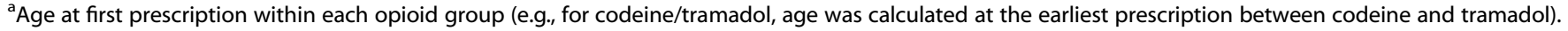

the interpretation of gene-level results, we additionally examined the direction of effect (i.e., risk or protective) from the SMMAT burden test output.

\section{RESULTS}

Among 10077 RIGHT participants, 2393 (24\%) participants were excluded because they were not prescribed any opioids or were not initiators of opioid use within the study date range (1/1/2005-12/31/ 2017). An additional 515 (6.7\%) participants were excluded due to self-reported non-white race and/or Hispanic ethnicity. The final sample included 7169 participants, of which 3636 (51\%) were prescribed codeine/tramadol, and 6649 (93\%) were prescribed hydrocodone/oxycodone. Additionally, 4284 participants were prescribed two or more opioids; $1069(25 \%)$ were prescribed opioids within 6 weeks of each other, and 784 (11\%) were prescribed opioids from different groups (e.g., codeine and oxycodone) within 6 weeks of each other. Participant characteristics are shown in Table 1.

\section{Association of variants with opioid response}

A total of 1940 common and low-frequency SNPs (MAF>0.01) were included in at least one analysis. Supplemental Fig. 4 displays the Manhattan plots representing pooled and sex-stratified analyses for all variants. Among participants prescribed hydrocodone/oxycodone $(n=6649)$, one intronic SNP was associated with a decreased risk of poor pain control in the pooled sample (rs76026520, MACROD2: $p$ value $=2.1 \times 10^{-5}, q$ value $=0.0397$ ). Among participants prescribed codeine/tramadol $(n=3636)$, two SNPs, one synonymous and one nonsynonymous, were associated with an increased risk of poor pain control in men (rs1056837, CYP1B1: $p$ value $=7.8 \times 10^{-6}, q$ value $=0.0075 ;$ rs 1056836, CYP1B1: $p$ value $=8 \times 10^{-6}, q$ value $=0.0075$, respectively). Among variants identified in CYP2D6, rs35742686 demonstrated the strongest association in any analysis, with the alternate allele dosage associated with decreased risk of adverse reactions to hydrocodone/oxycodone in the pooled sample ( $p$ value $=7.5 \times$ $10^{-5}, q$ value $=0.1436$ ). However, this association did not meet our statistical significance criterion. Table 2 summarizes the characteristics of these SNPs. A complete list of common variants associated with opioid response at the suggestive threshold of $p$ value $<0.001$ is summarized in Supplementary Table 2 .

\section{Association of genes with opioid response}

A total of 77 genes were included in at least one gene-level analysis in our study, and a $p$ value $\leq 0.05 / 77 \times 2$ (i.e. $3.2 \times 10^{-4}$; Bonferroni correction) was considered statistically significant among all tests. From these analyses, a total of seven genes were significantly associated with different opioid outcomes. Table 3 summarizes the genes associated with opioid response with the functional impact variant filter. The $F A A H$ gene was associated with an increased risk of adverse reactions to codeine/tramadol in the pooled sample $\left(p\right.$ value $\left.=2.0 \times 10^{-7}\right)$, and separately for men $(p$ value $=2.1 \times$ $10^{-6}$ ) and women $\left(p\right.$ value $\left.=1.0 \times 10^{-4}\right)$. The $F A A H$ gene was also associated with an increased risk of adverse reactions to hydrocodone/oxycodone in the pooled sample $\left(p\right.$ value $\left.=7.6 \times 10^{-5}\right)$, and this association was primarily driven by men $\left(p\right.$ value $\left.=1.4 \times 10^{-10}\right)$. In addition, the TYMS gene was associated with an increased risk of poor pain control to codeine/tramadol in the pooled sample ( $p$ value $=3.5 \times 10^{-5}$ ), and this association was primarily driven by men $\left(p\right.$ value $\left.=1.1 \times 10^{-6}\right)$. Finally, the SCN1A gene was associated with an increased risk of adverse reactions to codeine/tramadol in the pooled sample $\left(p\right.$ value $\left.=2.0 \times 10^{-4}\right)$, with the strongest association observed in women $\left(p\right.$ value $\left.=5.0 \times 10^{-4}\right)$.

Some associations were significant only among men. In particular, the NAT2 and CYP3A4 genes were associated with increased risk of adverse reactions to codeine/tramadol ( $p$ value $=2.0 \times 10^{-4}$ and $p$ value $=1.0 \times 10^{-4}$, respectively), and the CYP1A2 gene was associated with increased risk of poor pain control to codeine/ tramadol $\left(p\right.$ value $\left.=1.0 \times 10^{-4}\right)$. Finally, the SLC22A2 gene was also associated with increased risk of poor pain control to hydrocodone/ oxycodone in men $\left(p\right.$ value $\left.=9.3 \times 10^{-6}\right)$.

\section{DISCUSSION}

In this study, we investigated the effects of genetic variation on with adverse reactions and poor pain control to four commonly 


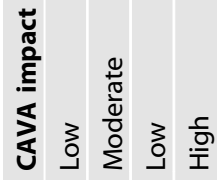

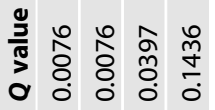

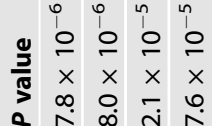

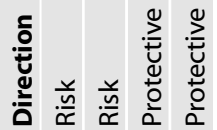

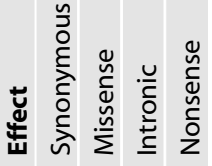

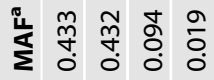

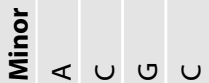

$\stackrel{\frac{.}{\circ}}{\frac{0}{\circ}} \cup v<ヒ$

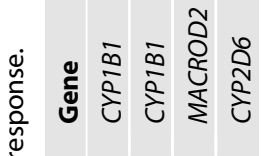

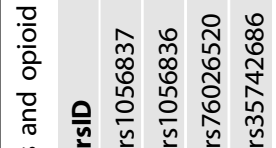

$\stackrel{\frac{n}{\pi}}{\frac{\pi}{6}}$

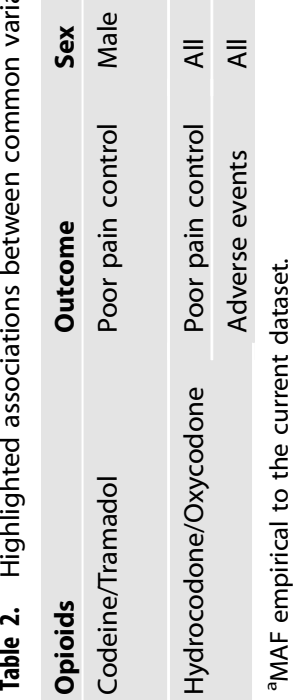

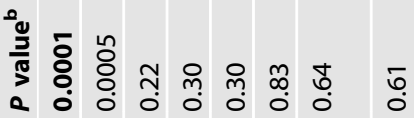

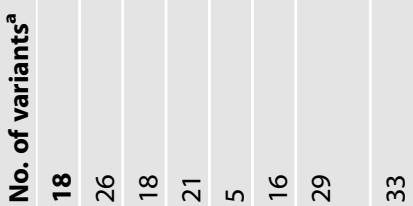

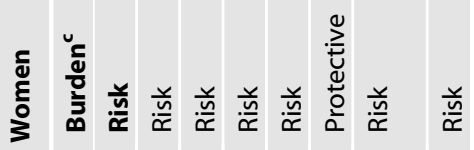

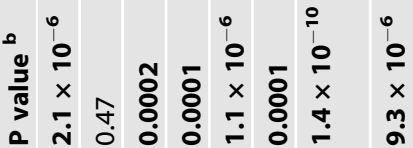

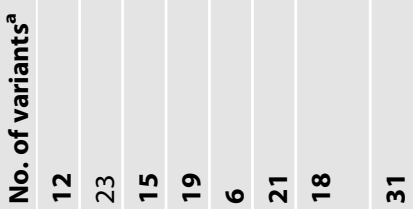

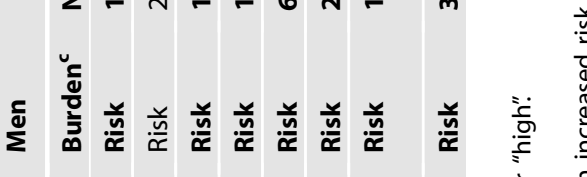

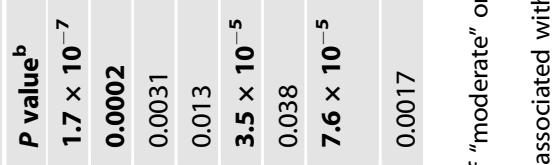
तै

फั0

"ِّ

ஸ்

2

i்

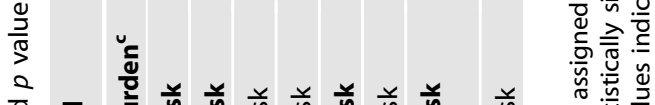

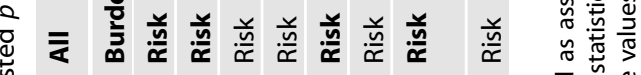

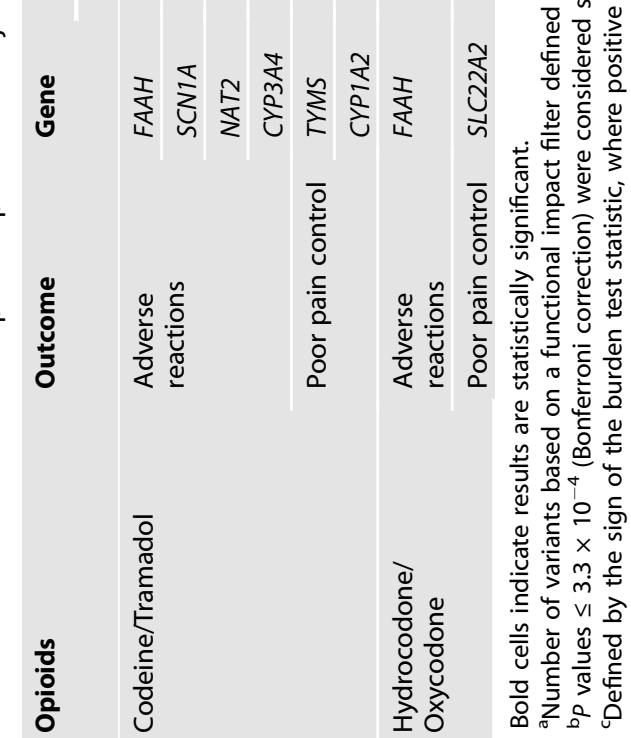


prescribed opioid medications. Common variants in MACROD2, CYP1B1, and CYP2D6 were associated with these outcomes. At the gene level, FAAH, SCN1A, and TYMS were also associated with these outcomes. Finally, we stratified our results by sex and found that NAT2, CYP3A4, CYP1A2, and SLC22A2 were associated with outcomes in men (but not in women).

\section{Common variants and opioid response}

CYP2D6 encodes an important metabolic enzyme that converts prodrugs-such as the opioids-to their active metabolites. Genetic polymorphisms in this gene impact enzyme activity, which can be classified as poor, intermediate, normal, or ultrarapid based on the effect of variants on the resulting protein structure and quantity. Among all CYP2D6 single-variant associations we examined, rs35742686 (p.R259Gfs*2, MAF $=0.018$ ) was suggestively associated with a decreased risk of adverse reactions to hydrocodone/oxycodone in our pooled cohort. This variant defines the CYP2D6*3 allele, which is inactive. Loss of function variants lead to reduced enzymatic activity and poor metabolism status [9]. Individuals with a CYP2D6 poor metabolizer phenotype -such as homozygotes of the CYP2D6*3 allele-have more limited conversion of prodrugs into their active metabolites, and therefore are less likely to experience adverse reactions relative to individuals with a CYP2D6 normal metabolizer phenotype [10, 16]. The marginal nature of the association in our study may be due to the relatively low minor allele frequency for this variant (MAF = 0.019) and therefore decreased power. Other CYP2D6 singlevariant associations examined included rs3892097 (c.506-1G > A, $M A F=0.20$ ), which defines the inactive $C Y P 2 D 6^{*} 4$ allele. We did not find significant associations for this variant across outcomes and cohorts (all $p$ values $\geq 0.05$ ). One possible explanation is the complex genetics of CYP2D6 metabolizer phenotypes, including copy-number states [29, 30], for which simple additive genetic models may inadequately capture genetic relationships with risk. Future research involving more complex copy-number and haplotype-based approaches are warranted.

We identified two variants associated with increased risk of poor pain control to codeine/tramadol among men in the CYP1B1 gene (rs1056837 and rs1056836). CYP1B1 encodes a cytochrome P450 monooxygenase involved in the metabolism of various endogenous substrates, including fatty acids, steroid hormones, and vitamins [31-35]. Similar to CYP2D6, CYP1B1 is involved in phase 1 drug metabolism. However, CYP1B1 is a relatively recently identified member of the CYP1 gene family and associations between this gene and opioid response are not yet established. Finally, one variant in the MACROD2 gene (rs76026520) was associated with increased risk of poor pain control to hydrocodone/oxycodone in the pooled sample. The MACROD2 gene has been associated with estrogen-independent growth and tamoxifen resistance in breast cancer patients [36], with obesity in the Korean population [37], and with autism spectrum disorder in the general population [38]. It is unclear whether MACROD2 plays a role in response to opioids.

\section{Association of genes with opioid response}

Variation in the FAAH gene has been previously associated with both pain perception and adverse reactions with use of morphine derivatives $[15,17]$. Consistent with the literature, $F A A H$ variation was associated with an increased risk of adverse reactions to both opioid groups. For codeine/tramadol, we observed this association in both men and women. For hydrocodone/oxycodone, this association was in the pooled sample but driven primarily by men. $F A A H$ (fatty acid amide hydrolase) encodes a serine hydrolase responsible for the degradation of fatty acid amide family of signaling lipids. FAAH targets include endocannabinoid ligands which regulate pain, inflammation, and several cognitive and emotional states (e.g., anxiety, depression) [39]. FAAH inhibitors have been shown to augment endocannabinoid signaling, thus reducing inflammation and producing pain relief comparable to tramadol [40]. Therefore, genetic variants leading to reduced FAAH protein function would be expected to exert a similar effect.

$F A A H$ variant rs324420 (p.Pro129Thr, $M A F=0.20$ ) is a wellcharacterized missense variant shown to lead to protein instability and decreased cellular $F A A H$ expression [41]. This variant has been associated with increased risk of refractory post-operative nausea/ vomiting, opioid-related respiratory depression [15], and increased risk for substance abuse [42]. We uncovered a significant genetic burden of the gene $F A A H$ associated with an increased risk of adverse reactions to codeine/tramadol (in men and women separately), and to hydrocodone/oxycodone (in the pooled sample, but driven primarily by men). However, we did not observe any association between opioid outcomes and individual FAAH SNPs, including variant rs324420. This may be due to the fact that variants with deleterious impact are more likely to be rare. For this reason, studies with increased sensitivity of biologically relevant results often perform both variant- and gene-level analyses.

The SCN1A gene was associated with an increased risk of adverse reactions to codeine/tramadol, and this association was driven primarily by women. The SCN1A gene is mainly expressed in cerebral tissue, and mutant SCN1A channels are highly heterogeneous. SCN1A has been associated with an increased risk of childhood seizures [43] and sudden infant death syndrome [44]. Future research may investigate associations between SCN1A phenotypes and opioid response in men and women. In addition, the TYMS gene was associated with an increased risk of poor pain control to codeine/tramadol, and this association was driven primarily by men. TYMS encodes for thymidylate synthase. Variants in the TYMS gene, which lead to increased thymidylate synthase expression, have been associated with poor response to methotrexate, an immunosuppressive drug $[45,46]$. It is unclear whether TYMS plays a role in the response to other drugs.

\section{Sex-specific associations of genes with opioid response}

We found that the NAT2 and CYP3A4 genes were associated with increased risk of adverse reactions to codeine/tramadol among men, but not women. NAT2 encodes an $\mathrm{N}$-acetyltransferase that activates and deactivates various exogenous drugs and carcinogens. Polymorphisms in NAT2 can lead to rapid, intermediate, and slow metabolizer phenotypes and are associated with drug toxicity [47]. The CYP3A4 gene is a member of the cytochrome P450 gene family, encoding for key enzymes involved in drug metabolism. Our finding is consistent with previous research documenting the role of CYP3A4 in the pharmacodynamics of opioids, including fentanyl, tramadol, and oxycodone $[48,49]$. Our sex-specific finding is also consistent with research indicating that sex is an important factor of CYP3A4 expression [50].

In addition, CYP1A2 was associated with increased risk of poor pain control to codeine/tramadol among men, but not women. CYP1A2 activity has been demonstrated to differ by sex [51], with estradiol being a contributing factor for this sex difference [52]. Finally, the SLC22A2 gene was associated with increased risk of poor pain control to hydrocodone/oxycodone among men, but not women. The SLC22A2 gene encodes for an important drug uptake transporter, and inhibition of SLC22A2 has been documented to affect morphine metabolism in mouse models [53]. However, more studies are needed to examine whether sex modifies the associations between SLC22A2 metabolizer phenotypes and opioid response.

Previous research suggested that interactions between sex and genetics on pain perception and opioid metabolism may be due to sex differences in the functioning of receptors (e.g., $\mu$-, $\delta$-opioid) and enzymes important to opioid pathways [54]. We have previously observed sex-specific associations between response to codeine and tramadol and CYP2D6 phenotypes [16]. However, Sadhasivam et al. [15]. documented SNPs associated with adverse reactions due to 
morphine administration in children, but the associations did not differ by sex. In addition, baseline pain sensitivity may affect opioidbased therapy effectiveness. Among candidate gene association studies, Kim et al [17]. reported variation in the $F A A H$ gene associated with cold pain sensitivity in European American men, but not in women.

PGX studies on opioids reporting sex-specific results are still scarce, despite NIH guidelines for clinical research stating the importance of sex as a biological variable to be factored in research design and statistical analysis [55]. The lack of sex-specific reports in PGx studies could lead to inappropriately broad assumptions on the effectiveness of pain therapies. Changes in clinical care based on these assumptions could negatively impact therapeutic response. Our findings add to a growing body of evidence indicating sexspecific genetic associations with opioid response.

\section{STRENGTHS, LIMITATIONS, AND CONCLUSION}

The strengths of this study include a large sample size and an ability to extract information directly from the EHR. In addition, we had an extensive PGx sequencing data and complete follow-up information after opioid prescription for all patients. However, there are several limitations. Patient outcomes collected retrospectively from EHRs are limited to those reported to a health care provider. Active, real-time follow-up of persons prescribed opioid analgesics may be needed to disentangle drug response from other factors influencing individuals' willingness to report outcomes to health care providers. In addition, $784(11 \%)$ of all prescribed patients were prescribed opioids from different groups (e.g., codeine and oxycodone) within 6 weeks of each other. Although an effort was made to associate outcomes to their corresponding opioids, some of the reported outcomes may have been due to other prescriptions or health events that occurred within 6 weeks of the prescription and were erroneously attributed to the opioid medication. Future research may be powered specifically to investigate PGX-wide associations with response to each opioid separately. Some patients may have not adhered to their opioid prescription, and we could not investigate the effects of use frequency or dosage on pain control and risk of adverse reactions. Type of adverse reactions and diagnosis associated with opioid prescription were not available for this cohort. Future research may investigate sex-specific associations between genetic variation and opioid response for specific adverse reactions or in cohorts of patients with specific diagnosis (e.g., oncological patients). The RIGHT protocol included a targeted panel of genes, and therefore our analysis did not permit investigation of genetic variation outside the capture design. Genome-wide association studies may reveal novel SNPs implicated in opioid response. In addition, many PGx genes exert phenotype via gene duplication and may be better uncovered using expression studies such as RNAseq. Finally, our cohort was from a non-Hispanic white population, and may not be generalizable to other populations.

In summary, we investigated sex-specific associations of PGx genes and their variants with response to commonly prescribed opioids in a large sample of individuals. We replicated previous findings that polymorphisms in some genes (e.g., CYP2D6, CYP3A4, CYP1A2) are related to opioid outcomes. We also identified novel genes for future studies of opioid response (e.g., TYMS, SLC22A2). Finally, our data suggest that there may be significant sex-based differences in the effects of these genes on opioid responses. Our findings highlight the importance of considering sex as a biological variable in the investigation of genetic associations with response to opioid analgesics.

\section{DATA AVAILABILITY}

For data sharing requests, please contact SJB at Bielinski.Suzette@mayo.edu.

\section{CODE AVAILABILITY}

All analyses were conducted using $\mathrm{R}$ version 3.6.2. Codes available upon request.

\section{REFERENCES}

1. Roehler DR, Hoots BE, Olsen EO, Kariisa M, Wilson NO, Rudd RA, et al. Annual surveillance report of drug-related risks and outcomes. United States, Atlanta, Georgia: Centers for Disease Control and Prevention (CDC); 2019.

2. Kalso E, Edwards JE, Moore RA, McQuay HJ. Opioids in chronic non-cancer pain: systematic review of efficacy and safety. Pain. 2004;112:372-80.

3. Abdel Shaheed C, Maher CG, Williams KA, Day R, McLachlan AJ. Efficacy, tolerability, and dose-dependent effects of opioid analgesics for low back pain: a systematic review and meta-analysis. JAMA Intern Med. 2016;176:958-68.

4. Becker JB, Chartoff E. Sex differences in neural mechanisms mediating reward and addiction. Neuropsychopharmacology. 2019;44:166-83.

5. Zorina-Lichtenwalter K, Meloto CB, Khoury S, Diatchenko L. Genetic predictors of human chronic pain conditions. Neuroscience. 2016;338:36-62.

6. Kumar S, Kundra P, Ramsamy K, Surendiran A. Pharmacogenetics of opioids: a narrative review. Anaesthesia. 2019;74:1456-70.

7. Young EE, Lariviere WR, Belfer I. Genetic basis of pain variability: recent advances. J Med Genet. 2012;49:1-9.

8. Ho KWD, Wallace MR, Staud R, Fillingim RB. OPRM1, OPRK1, and COMT genetic polymorphisms associated with opioid effects on experimental pain: a randomized, double-blind, placebo-controlled study. Pharmacogenomics J. 2020;20:471-81.

9. Gaedigk A, Ingelman-Sundberg M, Miller NA, Leeder JS, Whirl-Carrillo M, Klein TE, et al. The Pharmacogene Variation (PharmVar) Consortium: incorporation of the human cytochrome P450 (CYP) allele nomenclature database. Clin Pharmacol Ther. 2018;103:399-401.

10. Crews KR, Gaedigk A, Dunnenberger HM, Leeder JS, Klein TE, Caudle KE, et al. Clinical Pharmacogenetics Implementation Consortium guidelines for cytochrome P450 2D6 genotype and codeine therapy: 2014 update. Clin Pharmacol Ther. 2014;95:376-82.

11. Fullerton EF, Doyle HH, Murphy AZ. Impact of sex on pain and opioid analgesia: a review. Curr Opin Behav Sci. 2018;23:183-90.

12. Averitt DL, Eidson LN, Doyle HH, Murphy AZ. Neuronal and glial factors contributing to sex differences in opioid modulation of pain. Neuropsychopharmacology. 2019;44:155-65.

13. Wiech K. Deconstructing the sensation of pain: the influence of cognitive processes on pain perception. Science. 2016;354:584-7.

14. Mogil JS. Qualitative sex differences in pain processing: emerging evidence of a biased literature. Nat Rev Neurosci. 2020;21:353-65.

15. Sadhasivam S, Zhang X, Chidambaran V, Mavi J, Pilipenko V, Mersha TB, et al. Novel associations between FAAH genetic variants and postoperative central opioid-related adverse effects. Pharmacogenomics J. 2015;15:436-42.

16. Lopes GS, Bielinski SJ, Moyer AM, Black JL, Jacobson DJ, Jiang RX, et al. Sex differences in associations between CYP2D6 phenotypes and response to opioid analgesics. Pharmacogenomics Pers Med. 2020;13:71-9.

17. Kim H, Mittal DP, ladarola MJ, Dionne RA. Genetic predictors for acute experimental cold and heat pain sensitivity in humans. J Med Genet. 2006;43:e40.

18. Bielinski SJ, Olson JE, Pathak J, Weinshilboum RM, Wang L, Lyke KJ, et al. Preemptive genotyping for personalized medicine: design of the right drug, right dose, right time-using genomic data to individualize treatment protocol. Mayo Clin Proc. 2014;89:25-33.

19. Bielinski SJ, St Sauver JL, Olson JE, Larson NB, Black JL, Scherer SE, et al. Cohort profile: the right drug, right dose, right time: using genomic data to individualize treatment protocol (RIGHT Protocol). Int J Epidemiol. 2019;49:23-24k.

20. Olson JE, Ryu E, Johnson KJ, Koenig BA, Maschke KJ, Morrisette JA, et al. The Mayo Clinic Biobank: a building block for individualized medicine. Mayo Clin Proc. 2013;88:952-62.

21. Rocca WA, Grossardt BR, Brue SM, Bock-Goodner CM, Chamberlain AM, Wilson $\mathrm{PM}$, et al. Data resource profile: expansion of the rochester epidemiology project medical records-linkage system (E-REP). Int J Epidemiol. 2018;47:368-j.

22. Liu HBS, Sohn S, Murphy S, Wagholikar KB, Jonnalagadda SR, Ravikumar KE, et al. An information extraction framework for cohort identification using electronic health records. AMIA Summits TransI Sci Proc. 2013;2013:149-153.

23. Kocher JP, Quest DJ, Duffy P, Meiners MA, Moore RM, Rider D, et al. The Biological Reference Repository (BioR): a rapid and flexible system for genomics annotation. Bioinformatics. 2014;30:1920-2.

24. Munz M, Ruark E, Renwick A, Ramsay E, Clarke M, Mahamdallie S, et al. CSN and CAVA: variant annotation tools for rapid, robust next-generation sequencing analysis in the clinical setting. Genome Med. 2015;7:76.

25. St Sauver JL, Olson JE, Roger VL, Nicholson WT, Black JL, Takahashi PY, et al. CYP2D6 phenotypes are associated with adverse outcomes related to opioid medications. Pharmgenomics Pers Med. 2017;10:217-27. 
26. Marco CA, Plewa MC, Buderer N, Black C, Roberts A. Comparison of oxycodone and hydrocodone for the treatment of acute pain associated with fractures: a double-blind, randomized, controlled trial. Acad Emerg Med. 2005;12:282-8.

27. Trescot AM, Datta S, Lee M, Hansen H. Opioid pharmacology. Pain Physician. 2008;11:S133-53.

28. Chen HCM, Pham DT. GMMAT: generalized linear mixed model association tests. R package version 1.3.1. United States, Texas, Houston: The University of Texas Health Science Center at Houston; 2020.

29. Beoris M, Amos Wilson J, Garces JA, Lukowiak AA. CYP2D6 copy number distribution in the US population. Pharmacogenet Genomics. 2016;26:96-9.

30. Jarvis JP, Peter AP, Shaman JA. Consequences of CYP2D6 copy-number variation for pharmacogenomics in psychiatry. Front Psychiatry. 2019;10:432.

31. Mesaros C, Lee SH, Blair IA. Analysis of epoxyeicosatrienoic acids by chiral liquid chromatography/electron capture atmospheric pressure chemical ionization mass spectrometry using [13C]-analog internal standards. Rapid Commun Mass Spectrom. 2010;24:3237-47.

32. Badawi AF, Cavalieri EL, Rogan EG. Role of human cytochrome P450 1A1, 1A2, $1 \mathrm{~B} 1$, and $3 \mathrm{~A} 4$ in the $2-, 4-$, and 16alpha-hydroxylation of 17 beta-estradiol. Metabolism. 2001;50:1001-3.

33. Lee AJ, Cai MX, Thomas PE, Conney AH, Zhu BT. Characterization of the oxidative metabolites of 17 beta-estradiol and estrone formed by 15 selectively expressed human cytochrome p450 isoforms. Endocrinology. 2003;144:3382-98.

34. Chen H, Howald WN, Juchau MR. Biosynthesis of all-trans-retinoic acid from alltrans-retinol: catalysis of all-trans-retinol oxidation by human P-450 cytochromes. Drug Metab Dispos. 2000;28:315-22.

35. Choudhary D, Jansson I, Stoilov I, Sarfarazi M, Schenkman JB. Metabolism of retinoids and arachidonic acid by human and mouse cytochrome P450 1b1. Drug Metab Dispos. 2004;32:840-7.

36. Mohseni M, Cidado J, Croessmann S, Cravero K, Cimino-Mathews A, Wong HY, et al. MACROD2 overexpression mediates estrogen independent growth and tamoxifen resistance in breast cancers. Proc Natl Acad Sci USA. 2014;111:17606-11.

37. Kim HR, Jin HS, Eom YB. Association of MACROD2 gene variants with obesity and physical activity in a Korean population. Mol Genet Genomic Med. 2021;9:e1635.

38. Jones RM, Cadby G, Blangero J, Abraham L, Whitehouse AJO, Moses EK. MACROD2 gene associated with autistic-like traits in a general population sample. Psychiatr Genet. 2014;24:241-8.

39. Ahn K, Johnson DS, Mileni M, Beidler D, Long JZ, McKinney MK, et al. Discovery and characterization of a highly selective FAAH inhibitor that reduces inflammatory pain. Chem Biol. 2009;16:411-20.

40. Bhuniya D, Kharul RK, Hajare A, Shaikh N, Bhosale S, Balwe S, et al. Discovery and evaluation of novel FAAH inhibitors in neuropathic pain model. Bioorg Med Chem Lett. 2019;29:238-43.

41. Chiang KP, Gerber AL, Sipe JC, Cravatt BF. Reduced cellular expression and activity of the P129T mutant of human fatty acid amide hydrolase: evidence for a link between defects in the endocannabinoid system and problem drug use. Hum Mol Genet. 2004;13:2113-9.

42. Flanagan JM, Gerber AL, Cadet JL, Beutler E, Sipe JC. The fatty acid amide hydrolase $385 \mathrm{~A} / \mathrm{A}$ (P129T) variant: haplotype analysis of an ancient missense mutation and validation of risk for drug addiction. Hum Genet. 2006;120:581-8.

43. Freilich ER, Jones JM, Gaillard WD, Conry JA, Tsuchida TN, Reyes C, et al. Novel SCN1A mutation in a proband with malignant migrating partial seizures of infancy. Arch Neurol. 2011;68:665-71.

44. Brownstein CA, Goldstein RD, Thompson CH, Haynes RL, Giles E, Sheidley B, et al. SCN1A variants associated with sudden infant death syndrome. Epilepsia. 2018;59:e56-e62.

45. Krajinovic M, Costea I, Chiasson S. Polymorphism of the thymidylate synthase gene and outcome of acute lymphoblastic leukaemia. Lancet. 2002;359:1033-4

46. Lima A, Seabra V, Bernardes M, Azevedo R, Sousa H, Medeiros R. Role of key TYMS polymorphisms on methotrexate therapeutic outcome in Portuguese rheumatoid arthritis patients. PLoS ONE. 2014;9:e108165.

47. Hein DW, Doll MA, Fretland AJ, Leff MA, Webb SJ, Xiao GH, et al. Molecular genetics and epidemiology of the NAT1 and NAT2 acetylation polymorphisms. Cancer Epidemiol Biomark Prev. 2000;9:29-42.

48. Nielsen LM, Olesen AE, Branford R, Christrup LL, Sato H, Drewes AM. Association between human pain-related genotypes and variability in opioid analgesia: an updated review. Pain Pract. 2015;15:580-94.

49. Kummer O, Hammann F, Moser C, Schaller O, Drewe J, Krahenbuhl S. Effect of the inhibition of CYP3A4 or CYP2D6 on the pharmacokinetics and pharmacodynamics of oxycodone. Eur J Clin Pharmacol. 2011;67:63-71.

50. Wolbold R, Klein K, Burk O, Nussler AK, Neuhaus P, Eichelbaum M, et al. Sex is a major determinant of CYP3A4 expression in human liver. Hepatology. 2003:38:978-88.

51. Parkinson A, Mudra DR, Johnson C, Dwyer A, Carroll KM. The effects of gender, age, ethnicity, and liver cirrhosis on cytochrome P450 enzyme activity in human liver microsomes and inducibility in cultured human hepatocytes. Toxicol Appl Pharmacol. 2004;199:193-209.

52. Xie C, Pogribna M, Word B, Lyn-Cook L Jr., Lyn-Cook BD, Hammons GJ. In vitro analysis of factors influencing CYP1A2 expression as potential determinants of interindividual variation. Pharmacol Res Perspect. 2017;5:e00299.

53. Zhu P, Ye Z, Guo D, Xiong Z, Huang S, Guo J, et al. Irinotecan alters the disposition of morphine via inhibition of organic cation transporter 1 (OCT1) and 2 (OCT2). Pharm Res. 2018;35:243.

54. Doehring A, Kusener N, Fluhr K, Neddermeyer TJ, Schneider G, Lotsch J. Effect sizes in experimental pain produced by gender, genetic variants and sensitization procedures. PLoS ONE. 2011;6:e17724.

55. NIH. Inclusion of Women and Minorities as Participants in Research Involving Human Subjects. National Institution of Health; 2017. https://grants.nih.gov/ policy/inclusion/women-and-minorities.htm.

\section{AUTHOR CONTRIBUTIONS}

GSL: study design, data analysis, writing. JLL: genetics consultation, revision. SJB: data provider, epidemiology consultation, revision. SMA: data analysis, revision. YZ: abstractor, revision. DCC: abstractor, revision. AMM: genetics consultation, revision. DJJ: epidemiology consultation, revision. LW: data analysis, revision. RJ: data analysis, revision. JLS: epidemiology consultation, revision. NBL: study design, bioinformatics consultation, revision.

\section{FUNDING}

This work was supported by the Mayo Clinic Specialized Center of Research Excellent on Sex Differences, the Mayo Clinic Center for Individualized Medicine, the Robert D. and Patricia E. Kern Center for the Science of Health Care Delivery, and the National Institutes of Health grants [U19 GM61388 (The Pharmacogenomics Research Network), R01 GM28157, U01 HG005137, R01 GM125633, R01 AG034676 (The Rochester Epidemiology Project), U54 AG044170 (Sex-Specific Effects of Endocrine Disruption on Aging and Alzheimer's Disease)], and [U01 HG06379 and U01 HG06379 Supplement (The Electronic Medical Record and Genomics (eMERGE) Network)].

\section{COMPETING INTERESTS}

The authors declare no competing interests.

\section{ADDITIONAL INFORMATION}

Supplementary information The online version contains supplementary material available at https://doi.org/10.1038/s41397-022-00265-9.

Correspondence and requests for materials should be addressed to Guilherme S. Lopes.

Reprints and permission information is available at http://www.nature.com/ reprints

Publisher's note Springer Nature remains neutral with regard to jurisdictional claims in published maps and institutional affiliations.

\footnotetext{
Open Access This article is licensed under a Creative Commons Attribution 4.0 International License, which permits use, sharing, adaptation, distribution and reproduction in any medium or format, as long as you give appropriate credit to the original author(s) and the source, provide a link to the Creative Commons license, and indicate if changes were made. The images or other third party material in this article are included in the article's Creative Commons license, unless indicated otherwise in a credit line to the material. If material is not included in the article's Creative Commons license and your intended use is not permitted by statutory regulation or exceeds the permitted use, you will need to obtain permission directly from the copyright holder. To view a copy of this license, visit http://creativecommons. org/licenses/by/4.0/.
}

(c) The Author(s) 2022 